\section{Xenotransplantation von Zellen, Geweben oder Organen}

\author{
Wissenschaftliche Entwicklungen, ethische \\ Implikationen und gesellschaftliche Rele- \\ vanz
}

von Eva M. Neumann-Held, Europäische Akademie Bad Neuenahr-Ahrweiler GmbH

Die Europäische Akademie führt seit April 1998 ein zweijähriges Forschungsprojekt zum Thema Xenotransplantation durch. Ziel ist es, möglichst noch vor dem Eintritt dieser neuen Transplantationstechnologie in die klinische Phase eine umfassende Erörterung der wissenschaftlichen Möglichkeiten, Schwierigkeiten und der ethischen und gesellschaftlichen Implikationen der Xenotransplantation zu erarbeiten. Damit soll im Rahmen dieses Akademieprojektes ein Beitrag zu einer fachlich fundierten und ethisch begründbaren Urteilsbildung in Politik und Öffentlichkeit geleistet werden.

Unter dem Begriff Xenotransplantation versteht man die Übertragung von lebens- und funktionstüchtigen Zellen oder Zellverbänden (einschließlich ganzer Organe oder Körperteile) zwischen verschiedenen Spezies. Damit steht dieser Begriff dem der Allotransplantation (Übertragung zwischen genetisch verschiedenen Individuen derselben Spezies) gegenüber.

Allotransplantationen sind selbst in bezug auf den Ersatz vollständiger Organe eine heutzutage nahezu routinemäßig durchgeführte medizinische Maßnahme, die zum Erhalt oder zur Erleichterung menschlichen Lebens eingesetzt wird. Ein nach wie vor großes Problem liegt aber darin, daß das Angebot an menschlichen Spenderorganen bei weitem nicht ausreicht, um dem Bedarf zu entsprechen. Da nicht für alle menschlichen Organe bei chronischem Versagen andere lebenserhaltende Maßnahmen zur Verfügung stehen, ist der "Tod auf der Warteliste" für ca. ein Drittel der Transplantationsbedürftigen immer noch die Regel. Erschwerend tritt hinzu, daß bei dem derzeitigen Organmangel die Indikationen für eine Transplantation sehr streng gestellt werden müssen, so daß der angemeldete Organbedarf eher noch zu niedrig angesetzt ist.
Neben der Weiterentwicklung der Allotransplantation sucht die Forschung daher verstärkt nach zusätzlichen Organquellen. Hierzu gehört neben der Entwicklung künstlicher Gewebe und Organe und der regenerativen Organogenese die Xenotransplantation.

Eine funktionierende Xenotransplantationstechnik könnte dazu dienen, menschliche Zellen, Gewebe oder Organe zu ersetzen. Es gibt sogar experimentelle Hinweise, daß in manchen Situationen ein Xenotransplantat gegenüber einem Allotransplantat die vorzuziehende Option sein könnte, wenn nämlich das Xenotransplantat gegen bestimmte menschliche Krankheitserreger resistent wäre. Xenotransplantate könnten aber auch "Überbrückungsfunktionen" ausüben. Hierbei würde man zum einen an einen Einsatz in den Situationen denken, in denen bei akutem Versagen des Eigenorgans ein Xenotransplantat so lange angeschlossen werden würde, bis ein geeignetes menschliches Spenderorgan zur Verfügung stünde (z.B. Herz). Zum anderen könnten Xenotransplantate zur vorübergehenden Entlastung der Eigenfunktionen eines erkrankten Organs dienen, um diesem so die Möglichkeit zur Regeneration zu geben (z.B. Leber).

In beiden Fällen könnte das Xenotransplantat später entfernt werden, wodurch notwendige Immunosuppressionsmaßnahmen entweder erheblich reduziert (bei Austausch durch ein menschliches Spenderorgan) oder ganz abgesetzt werden könnten (nach Regeneration des Eigenorgans).

Eine realistische Einschätzung dieser Hoffnungen und Erwartungen an die Xenotransplantation erfordert es, sich mit den Problemstellungen und möglichen Risiken zu beschäftigen, die sich im Umfeld der Xenotransplantationstechnik und -methodik ergeben können. Hier zeigt sich, daß eine umfassende Beurteilung nur auf der Basis umfangreicher Kenntnisse aus diversen biologischen und medizinischen Disziplinen erfolgen kann. Deren Ergebnisse müssen dann, wenn es um die normative Frage nach dem Umgang mit Xenotransplantation geht, zum Gegenstand juristischer und philosophisch-ethischer Reflexion werden.

Ein kurzer Blick auf die bisher gewonnenen empirischen Erkenntnisse läßt den schnellen Übergang der Xenotransplantationstechnik in die klinische Phase zumindest in bezug auf 
die Transplantation vollständiger Organe sehr fraglich erscheinen.

Während die Transplantation von Zellen und Geweben zwischen artverschiedenen Tieren und zum Teil auch schon vom Tier auf den Menschen inzwischen erste Erfolge zeitigt, verläuft diejenige ganzer Organe bisher gänzlich unbefriedigend. Eine der Ursachen hierfür ist in hyperakuten Abstoßungsreaktionen zu sehen. Ein Ansatz zur Beherrschung hyperakuter Abstoßungsreaktionen besteht in massiver Immunosuppression. Diese radikale Unterdrükkung der Reaktivität des Immunsystems ist aber letztlich letal aufgrund der erhöhten Infektanfälligkeiten und ist daher, zumindest als alleinige Maßnahme, keine Lösung des Problems. Eine andere Strategie wäre die Züchtung und Klonierung von Spendertieren, die gentechnisch so zu verändern wären, daß ihre Organe beim Menschen keine Abstoßungsreaktionen mehr hervorrufen. Forschungen in dieser Richtung stehen noch am Anfang. Sie sind jedoch noch nicht auf die zusätzlichen Probleme physiologischer Unverträglichkeit gerichtet und es ist noch nicht geklärt, inwieweit physiologische Unterschiede zwischen Spendern und Empfängern eine erfolgreiche Xenotransplantation ermöglichen könnten.

Darüber hinaus könnte die Transplantation über Artgrenzen hinweg zu einer Übertragung tierischer Viren auf den Menschen führen, was u.a. eine Rekombination zwischen tierischen und menschlichen Viren zur Folge haben könnte. Die noch zu analysierenden Möglichkeiten der Virusinfektion und -rekombination und die damit verbundene Gefahr der Ausbreitung neuer Viren verweist unmittelbar auch auf die ethische und rechtliche Dimension der Xenotransplantation. Wenn auch eine wissenschaftliche Einschätzung dieser Gefahr durch die Virologie erfolgt, so muß die Bewertung des möglichen Risikos für ganze Bevölkerungsgruppen $\mathrm{Ge}$ genstand ethischer und rechtlicher Betrachtungen sein. Darüber hinaus ist eine Analyse und Bewertung der tierethischen und tierrechtlichen Problematik erforderlich sowie die Aufdeckung eines möglichen Regelungsbedarfes, der in der bestehenden Gesetzgebung nicht erfaßt ist.

In der Projektgruppe Xenotransplantation der Europäischen Akademie soll, ausgehend von einer Analyse des naturwissenschaftlichen, ethischen und rechtlichen Forschungs- und
Diskussionsstands zur Xenotransplantation, eine wechselseitige Durchdringung von wissenschaftlicher Diskussion und ethischer bzw. rechtlicher Analyse im Hinblick auf den Untersuchungsgegenstand erreicht werden. Die für die Analyse der Probleme im Umfeld der Xenotransplantation erforderliche Transdisziplinarität wird in der Projektgruppe durch ihre $\mathrm{Zu}$ sammensetzung aus Experten der Transplantationsmedizin, Tiermedizin, Virologie, Immunologie und Genetik einerseits und der Philosophie und Jurisprudenz andererseits gewährleistet.

Ziel der Projektarbeit ist die Erstellung eines transdisziplinären Memorandums, welches die in Deutschland eben erst beginnende Debatte um die Xenotransplantation sachlich fundieren und strukturieren hilft und an die internationale Diskussion anbindet. Darüber hinaus soll ein Beitrag zu gesellschaftlichen Prozessen der Entscheidungsfindung geleistet werden, in denen es um die Frage geht, wie der weitere Umgang mit der Xenotransplantation zu beurteilen ist und ob sie gegebenenfalls die Erarbeitung spezieller Richtlinien erforderlich macht.

\section{Projektgruppe}

Vorsitz: Prof. Dr. phil. J.P. Beckmann, Institut für Philosophie, FernUniversität Hagen

Prof. Dr. med.vet. Dr.med.vet.habil. Dr. h.c. G. Brem, Institut für Tierzucht und Genetik, Veterinärmedizinische Universität Wien

Prof. em. Dr. med. F.W. Eigler, Abteilung für allgemeine Chirurgie, Universitätsklinik Essen

Prof. Dr. med. Dr. rer.nat. habil. W. H. Günzburg, Institut für Virologie, Veterinärmedizinische Universität Wien

Prof. Dr. med. Dr. med. vet. C. Hammer, Institut für Chirurgische Forschung, Klinikum Großhadern b. München

Prof. em. Dr. med. Dr. med. dent. Dr.h.c. W. Müller-Ruchholtz, Institut für Immunologie, Klinikum der Universität Kiel

Prof. Dr. iur. Dr. h.c. H.L. Schreiber, Juristisches Seminar der Universität Göttingen 


\section{Kontakt}

Dr.rer.nat.E.M. Neumann-Held

Europäische Akademie zur Erforschung von Folgen wissenschaftlich-technischer Entwicklungen

Bad Neuenahr-Ahrweiler GmbH

Postfach 1460, D-53459 Bad Neuenahr-Ahrweiler

Tel.: ++ 49-(0) 2641/754-310

Fax: ++ 49-(0) 2641/754-320

E-mail: eva.neumann-held@dlr.de

》

\section{Technikfolgenabschätzung und Ethik}

Eine Untersuchung zu Verwendung und Stellenwert ethischer Kriterien in Konzepten und Verfahren zur Technikfolgenabschätzung anläßlich von Innovationen in der modernen Bio- bzw. Gentechnologie

von Barbara Skorupinski und Konrad Ott, Institut für Sozialethik der Universität Zürich

Im Rahmen eines Forschungsprojektes am Institut für Sozialethik der Universität Zürich soll am Beispiel der modernen Bio- und Gentechnologie zur Klärung des Verhältnisses von TA und Ethik beigetragen werden. Dieser Klärung wird für die konzeptionelle Weiterentwicklung von TA große Bedeutung beigemessen.

Technikfolgenabschätzung (TA) ist als eine politiknahe Institution entstanden, mit deren Hilfe verantwortliche Entscheidungen über wissenschaftliche und technische Entwicklungen ermöglicht werden sollen. Seit ihrer Entstehung hat TA verschiedene Veränderungen und Differenzierungen erfahren. Eine dieser Veränderungen besteht in der wachsenden Bedeutung der Bewertungsdimension. TA kommt die Aufgabe $\mathrm{zu}$, Orientierungswissen für politische Entscheidungen unter den Bedingungen von Risiko und Ungewißheit bereitzustellen. Sofern innerhalb von TA-Verfahren Aussagen über die Erwünschtheit oder Unerwünschtheit technologischer Innovationen formuliert werden, wird implizit oder explizit auf Werte oder Normen zurückgegriffen. Daher besteht eine begründungstheoretische Verbindung zwischen TA und
Ethik. Dabei werden zunehmend die diesbezüglichen Defizite der ursprünglichen Konzeption deutlich.

Diese Defizite lassen sich in folgende Fragen kleiden:

- Auf welche Weise können TA-Konzeptionen und -Verfahren der Wertdimension angemessen Rechnung tragen?

- Wie können Werturteile in TA-Verfahren begründet und kritisiert werden?

- Wie verhalten sich ethisch relevante Argumente $\mathrm{zu}$ anderen vorgebrachten Gründen?

- Wie gehen Werturteile in Szenarien und Empfehlungen ein?

Eine systematische Klärung des Verhältnisses zwischen Technikfolgenabschätzung und Ethik ist daher für die konzeptionelle Weiterentwicklung von TA von großer Bedeutung.

In unserem Forschungsprojekt sollen - als Beitrag zu eben dieser Klärung - die folgenden Thesen begründet und daraus Gesichtspunkte und kritische Maßstäbe entwickelt werden, die für Diskurse im Bereich moderner Bio- und Gentechnologien relevant sind.

1. TA ist aus prinzipiellen Gründen nicht von ethischen Fragen ablösbar. Konzeptionelle Probleme von TA sind immer mit normativen Fragen verknüpft.

2. Wenn TA-Verfahren zu Ergebnissen kommen wollen, die über die bloße Präsentation von Optionen hinausgehen, ist es unerläßlich, der Bewertungdimension zentrale Bedeutung innerhalb von TA-Verfahren zukommen zu lassen.

3. Es ist möglich und sinnvoll, Konzepte zu diskursiven und partizipativen TA-Verfahren in der Diskursethik zu verankern. Diese bedarf in bezug auf diskursive und partizipative TA-Verfahren jedoch der Spezifikation bzw. Modifikation.

4. Es ergibt sich die Notwendigkeit, partizipative Elemente in TA-Konzeptionen bzw. Verfahren zu integrieren.

Das Forschungsprojekt versucht die Begründung dieser Thesen aus zwei Richtungen. Zum einen in einem deskriptiv-empirischen, zum anderen in einem ethisch-normativen Zugang. Der empirisch-deskriptive Zugang umfaßt eine 